\title{
Effect of the Local Atomic Ordering on the Stability
}

\section{of $\beta$-Spodumene}

\author{
Radhika L. Moore, Brian S. Haynes and Alejandro Montoya ${ }^{*}$
}

School of Chemical and Biomolecular Engineering

Faculty of Engineering and Information Technologies, The University of Sydney,

Sydney, NSW 2006, Australia

* Corresponding Author: E-mail: Alejandro.montoya@sydney.edu.au

Phone: +61293512040

Fax: +61293512845 


\section{SUPPORTING INFORMATION}

In this document we provide results illustrating the accuaracy of the PBEsol functional to predict the heat capacity and entropy of $\alpha$ - spodumene and $\beta$-spodumene. The close agreement between the theoretical and experimental values indicates that the PBEsol functional is able to accurately predict thermodynamic parameters of spodumene polymorphs.

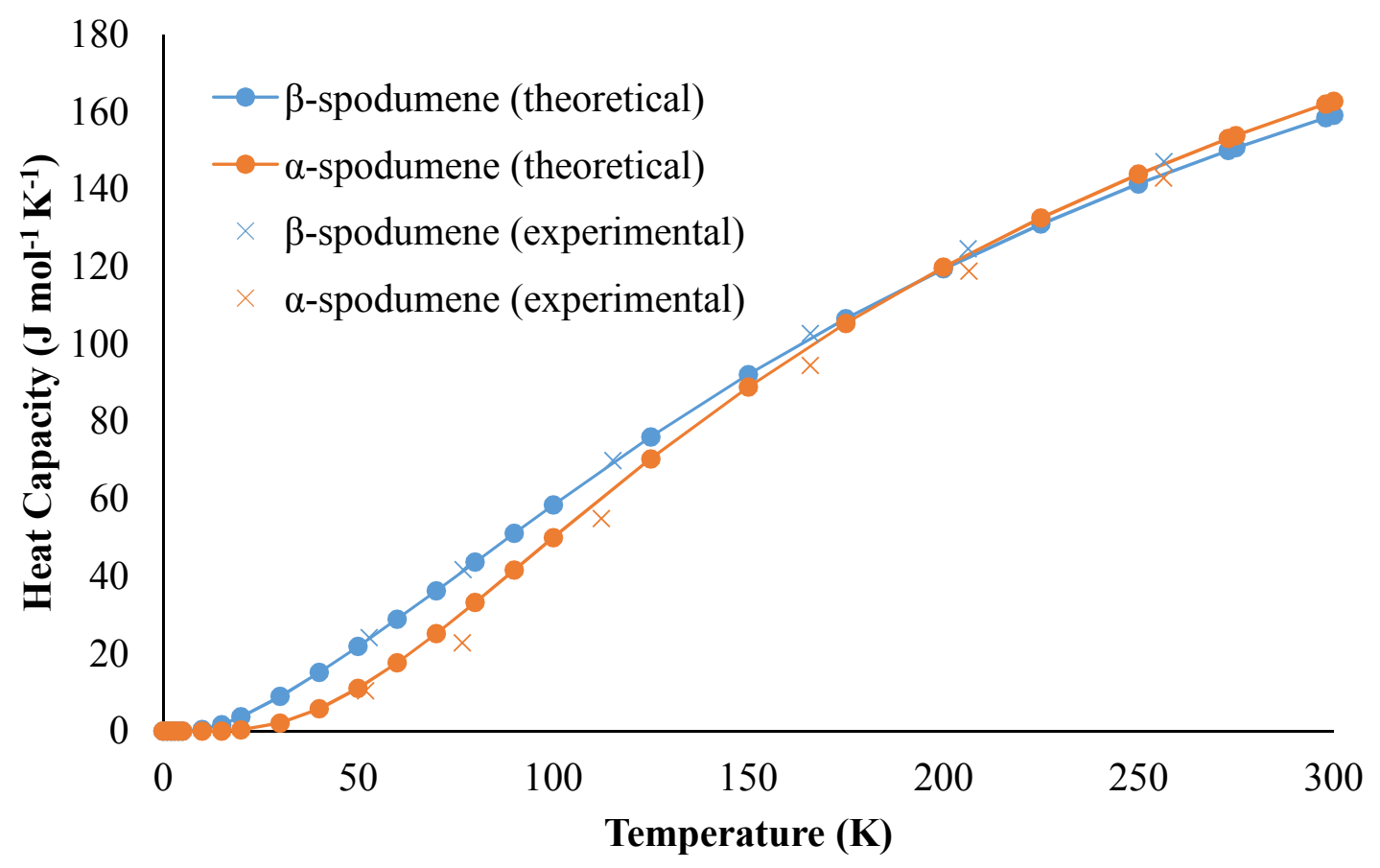

Figure S1. Specific heat capacity $\left(\mathrm{J} \mathrm{mol}^{-1} \mathrm{~K}^{-1}\right)$ of $\alpha$-spodumene and $\beta$-spodumene as a function of temperature. The theoretical heat capacity was obtained with the PBEsol functional and the experimental values were taken from literature. ${ }^{1}$ The electronic energy and the phonon density of states of a relaxed $\beta$-spodumene crystal structure were used to obtained the theoretical values. 


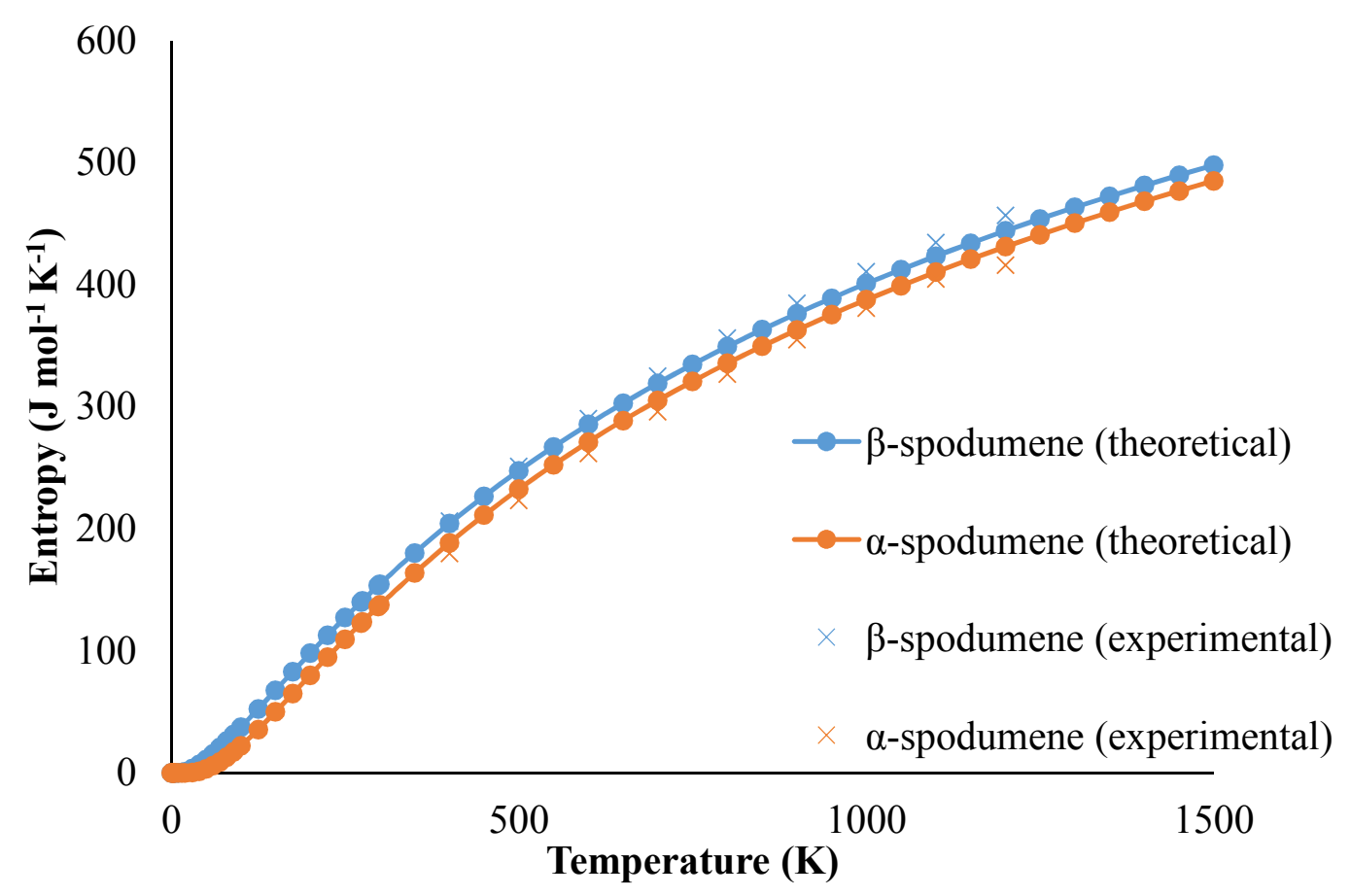

Figure S2. Entropy $\left(\mathrm{J} \mathrm{mol}^{-1} \mathrm{~K}^{-1}\right)$ obtained with the PBEsol functional of $\alpha$ - spodumene and $\beta-$ spodumene as a function of temperature. The electronic energy and the phonon density of states of a relaxed $\beta$-spodumene crystal structure were used to obtained the theoretical values and the experimental values were taken from literature. ${ }^{1}$

(1) Pankratz, L. B.; Weller, W. W. "Thermodynamic properties of three lithiumaluminum silicates," United State Department of the Interior: Bureau of Mines, 1967. 